\title{
Dynamic Grouping Frame-slotted Aloha
}

\author{
Mian Hammad Nazir \\ Department of Electrical Engineering, Jonkoping \\ University, Sweden
}

\author{
Nathirulla Sheriff \\ Department of ECE, \\ Sri Krishna College of Technology, India
}

\begin{abstract}
Passive UHF RFID systems using Dynamic Frame-Slotted ALOHA (DFSA) adjust the frame size according to the number of tags, but frame size $\mathrm{N}$ is equals to $\mathbf{2}^{\boldsymbol{Q}}$ and cannot be adjusted exactly to the number of tags to be identified. In this paper, we proposed a new Aloha based anti-collision algorithm which divides the tags and adjusts the frame sizes in such a manner as to always keep the system efficiency optimal. The algorithm is also designed in order to reduce the identification time for identifying the total number of tags. As a result the proposed algorithm uses minimum number of slots to read the tags with minimum identification time. Simulation results show that the algorithm gives maximum slot efficiency with every frame maintaining the system efficiency close or equal to $36.8 \%$ compared to other anti collision algorithms.
\end{abstract}

\section{Keywords}

Passive UHF RFID, Anti -collision, EPC class 1 Gen 2, grouping approach, maximum system efficiency

\section{INTRODUCTION}

RFID (Radio Frequency Identification) is a technology which uses radio waves in order to identify several objects. Mainly in RFID system we have two main components, the reader (sometimes called as an interrogator) and the Tags. When a tag enters the RF field, the reader asks an interrogating question (e.g. "what is your identity?"). The tag then responds with an answer to the reader (e.g. I am product XYZ).In the similar way like other identifying technologies like bar codes, RFID systems allow objects to be identified.

In RFID systems there can be three types of collisions [1] namely reader to reader collision in which multiple tags hear from the same reader at the same time, tag to tag collision in which multiple tags respond to the same reader at the same time and reader to tag collision in which signals from the neighbouring reader interfere with the tag's response signal being received at another reader.

In this paper we will focus on tag to tag collision and how to resolve this using an efficient anti collision algorithm.Several multi tag passive RFID anti-collision algorithms have been proposed and among them the most widely used are frame slotted ALOHA (FSA) algorithms and binary search tree algorithms. FSA are more widely used due to their simplicity and easy to understand feature and more efficient results compared to the Tree based.

ISO 18000-6 protocol has three types A,B,C. Type A and type $\mathrm{C}$ adopt ALOHA-based anti-collision schemes, type $\mathrm{B}$ adopts a tree-based scheme. The latest RFID standard announced by IEC and later on accepted by ISO is ISO/IEC 18000- 6C which is also known as UHF Gen 2 [2].
This paper presents an enhanced method for frame size estimation which is purely based on Q-algorithm. The proposed algorithm is very similar to $\mathrm{Q}$ algorithm but instead shows far better performance. The algorithm uses an approach to randomly participate in to the group to be identified in the coming frame and $\mathrm{Q}$ control and grouping during the frame. It first calculates the probability of the collision $\left(P_{\text {coll }}^{N, n}\right)$ and compares this value with the threshold collision value $\left(P_{\text {coll }}^{N, n}\right)$ which is probability of collision under optimal condition (number of tags= number of slots) that is fixed for each frame and is calculated by the reader, if the condition $\left(P_{\text {coll }}^{N, n}>P_{\text {coll }}^{N, n}{ }_{T h}\right)$ becomes true the algorithm divides the tags among groups using dynamic grouping approach in which after each frame tags are divided and identified as result of which all the tags to be identified participate in all the grouping activity. This scheme is better than static grouping scheme. The simulation results show that the proposed algorithm with dynamic grouping approach and intelligent frame sizing reduce the total number of slots for DFSA and identification time for UHF Gen2 based protocol.

The rest of the paper is organized as follows. Section II shows a brief introduction to EPC-global Class 1 Gen 2 protocol along with Q algorithm so to compare with proposed scheme. In Section III we propose the dynamic grouping and optimal frame sizing approach. The performance analysis and simulation results are presented in Section IV and finally the conclusion under Section V.

\section{EPC GLOBAL CLASS -1 GENERATION 2 AND Q- ALGORITHM}

It is also commonly known as the "Gen 2" standard, this standard defines the physical and logical requirements for a passive-backscatter, reader-talks-first RFID system operating in the $860 \mathrm{MHz}-960 \mathrm{MHz}$ frequency range. Many optional features have been added in this new standard. EPC Class 1 Gen 2 uses Dynamic frame slotted Aloha (DFSA) [3]-[9] based on Q- algorithm. The Inventory operations are based on slotted Aloha collision resolution. The reader issues a 22 bit QUERY command, and each tag randomly selects a number with a range between 0 to frame size -1 . A tag that rolls a 0 , replies immediately by issuing a 16 bit ID, RN16; all tags that roll other numbers record those numbers in a counter and wait for their turn. If a tag successfully transmits its RN16 without error or collision, the reader sends 18 bit ACK (RN16) to the tag After receiving ACK from the reader the tag sends the data including 96 or 256-bit Electronic Product Code (EPC) and 16-bit CRC. The reader, after either receiving a reply or no response, can issue a 4 bit QUERY REP command, causing all the tags to decrement their counters by 1 ; any tag reaching a counter value of 0 responds as shown in Figure.1. 


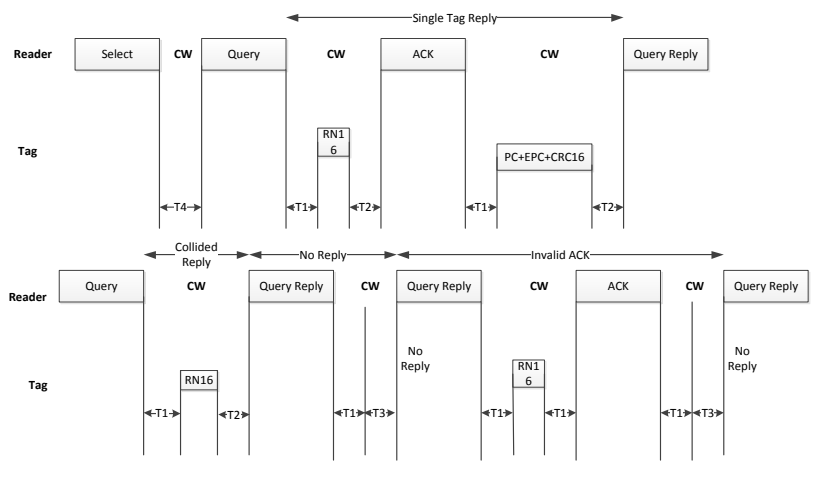

Figure 1: Example of EPC Class 1 Gen 2 Protocol

Frame sizing is the most important part of DFSA with Qalgorithm in EPC-global class 1 Gen 2 but there are some negative sides of this protocol like, frame size cannot be linearly adjusted, an inventory round is equal to a complete set of $2^{Q}$ slots which means the $Q$ cannot be changed even if we find extreme collision or extreme empty slots so that is why system efficiency decreases and identification time increases. The Q-Algorithm introduced in EPC-global Class 1 Gen 2 protocol is very simple approach for DFSA unlike other methods with compact representation of $\mathrm{N}=2^{Q}$ with early end of slots using Query Reply and dynamic adjustment of Q by Query or Query Adjust compare to estimators in that requires complicate computations or large memory.

\subsection{The Proposed Optimal dynamic frame slotted aloha (DGFSA)}

In this section, we propose an enhanced method which uses dynamic grouping approach in which tags are divided and grouped and one group is identified in one frame and then again divided and grouped and identified in the next frame. All the tags to be identified participate in all the grouping activity. This method is better than static grouping approach in which grouping is done and then tags are identified group by group. Dynamic grouping approach is better as the tags divided and grouped after every frame best fit according to the frame size keeping optimal system efficiency. The algorithm [8] use static grouping approach in which the same $\mathrm{Q}$ for every frame is decided according to the number of tags in a group and while in DGFSA Q is decided at the start of each frame according to the number of tags in that group so the novelty of grouping is maintained.

In DGFSA the reader divides the total number of tags by frame size at the start of every frame which will give some fractional value. The total number of tags are again divided by this fractional value which gives the optimal number of tags $(\mathrm{n}=\mathrm{N})$ which will be called as group 1 and the rest of tags are called as group 2 and so on .The tags under group 2 and after will be read in second read cycle and so on respectively, but the same process of division will also be applied on them which will again result in the one group with optimal number of tags and rest of the tags will come after in the next group. This process is repeated until all the tags have been identified. The detailed description of DGFSA is given in the following sub sections.

\subsubsection{Mathematical Analysis of DGFSA}

The performance of the system (system efficiency) is greatly degraded when there are large number of tags compared with the frame size, early research was done in order to increase the frame size according to the number of tags, but frame size has limitations so when there are unlimited number of tags than number of tags also need to be adjusted along with frame size.

The binomial distribution function describing $\mathrm{n}$ tags transmitting there ID to a slot is given in Equation (5).

$$
\mathrm{P}_{\mathrm{r}_{\mathrm{n}, \mathrm{p}}}(X=r)=\left(\begin{array}{l}
n \\
\mathrm{r}
\end{array}\right) \cdot p^{r}(1-p)^{n-r}
$$

Equation (1) can be used in order to derive the successful Probability of the slot with one tag among each slot, as given in equation (2).

$$
\mathrm{a}_{1}^{\mathrm{N}, \mathrm{n}}=\mathrm{P}_{\mathrm{r}_{\mathrm{n}, \mathrm{p}}}(X=1)=n \cdot p(1-p)^{n-1}
$$

Equation (2) the condition under which the probability of success full slots will be maximum i.e. system efficiency will be maximum.

$$
\frac{\mathrm{d}\left(\mathrm{a}_{1}^{\mathrm{N}, \mathrm{n}}\right)}{\mathrm{dn}}=n \cdot(1-p)^{n-1}-n \cdot(n-1) \cdot p(1-p)^{n-2}=0 \text { (3) }
$$

From Equation (3) optimal condition is obtained, $\mathrm{p}=1 / \mathrm{n}$. Let the frame size be $\mathrm{N}$ than under the optimal condition we have the following relation.

$$
\mathrm{N}=\mathrm{n}
$$

Relation (4) is used to calculate the probability of collision, idle and successful slots under optimal condition which are shown in equations respectively.

$$
\begin{aligned}
& P_{\text {idl }_{T h}}=\left(1-\frac{1}{n}\right)^{n} \\
& P_{\text {Suc }_{T h}}=\left(1-\frac{1}{n}\right)^{n-1} \\
& P_{\text {col }_{T h}}=1-\left(1-\frac{1}{n}\right)^{n}-\left(1-\frac{1}{n}\right)^{n-1}
\end{aligned}
$$

These equations give the optimal probability values which are fixed for each frame size and provide the required threshold values.

$$
\begin{aligned}
P_{\text {idle }} & =\left(1-\frac{1}{N}\right)^{n} \\
P_{\text {succ }} & =\left(\frac{n}{N}\right) \cdot\left(1-\frac{1}{N}\right)^{n-1} \\
P_{\text {coll }} & =1-\left(1-\frac{1}{N}\right)^{n}-\left[\left(\frac{n}{N}\right) \cdot\left(1-\frac{1}{N}\right)^{n-1}\right]
\end{aligned}
$$

Equations (8-10) give the probability of idle, successful and collision slots with respect of different values of $n$ and $N$. Figure1 shows the complete model of DGFSA, which is purely based on $\mathrm{Q}$ algorithm in order to keep in consideration all the delays due to inventory rounds.

\subsubsection{Dynamic Tag grouping and Frame Sizing by Reader}

Figure 3 shows how dynamic grouping and frame sizing is done by the reader. As the number of tags increases with respect to the frame size the probability of collision slots to occur also increases, Figure 3 shows that if $P_{\text {coll }}^{N, n}>P_{\text {col }}^{N, n}$ than 
the reader will divide the total number of unread tags by frame size

$$
m=\frac{\text { Total number of unread tags }}{\text { Frame Size }}
$$

Where $P_{\text {coll }}^{N, n}$ is the probability of collision when number of tags is greater than frame size and $P_{\text {coll }}^{N, n}$ is the probability of collision under optimal condition $(n=N)$ and this value is always constant for every frame irrespective of frame size $\left(P_{\text {coll }}^{N, n}=0.2640\right)$.

So if the number of tags is greater than size of the frame than the condition $P_{\text {coll }}^{N, n}>P_{\text {coll }}^{N, n}$ will become true and the reader will divide the tags in to groups by using relation (11). This relation will give the results in fractional value. In the next step the reader will divide total number of tags with this fractional value which will give optimal number of tags, here in group 1 and the rest of the tags will be identified in the next read cycle.

In Figure 2, Step 2 shows that how many inventory rounds are saved, the reader divides the fractional result obtained from relation (11) by 2 till its value is $\geq 2$. In this way if there are very large number of tags (e.g.; $n=1500$ ) than despite of dividing them in to frame size of (e.g.; $\mathrm{N}=256$ ), we can divide them among two groups of 512, so saving inventory rounds.

The above process can be well understood with the example; if the initial frame size is 256 and number of unread tags are 1500 than the probability of collision slots to occur in a frame will be greater than the threshold value than the reader will perform the following operations under these steps.

\section{Step 1}

$$
m=\frac{1500}{256}=5.859375
$$

Step 2

Here if $(m \geq 4)$ is used, which if satisfied than the reader divides ' $m$ ' by 2 till the result reaches $\geq 2$, in this case it will be like

$$
m=\frac{5.859375}{2}=2.9296875
$$

Step 3

$$
n^{\prime}=\frac{1500}{2.9296875}=512:: \mathrm{n}^{\prime} \text { is no. of tags in }
$$

First frame (group 1)

Step 4

$$
2^{Q}=n^{\prime}, Q=\frac{\ln n^{\prime}}{\ln 2}=\frac{\ln 512}{\ln 2}=9.0
$$

The reader selects the number of tags after computation as shown under step 3 using select command [2], SELECT selects the particular tag population based on user defined criteria. From step 4 readers will calculate the value of $\mathrm{Q}$ which can be sent to the tags (The SEL and TARGET) fields inside query command choose which tags respond to query. The total number of the tags that will participate in the next frame will be calculated as,

$n=\left(n^{\prime}-N_{\text {succ }}\right)+$ Remaining tags which didn't participate
Where $\mathrm{N}_{\text {succ }}$ represents tags successfully identified in inventory round .Now the remaining number of tags i.e.; 1311 will be covered in the second read cycle. As the number of tags is again larger than frame size i.e.; 512 that was set in the previous frame, again the reader will perform all the four steps and again the tags that will be identified in this read cycle will be 512 and frame size calculated is 512 , so efficiency of this read cycle will also be optimal.

The remaining number of tags are 1122, and frame size is 512 the remaining number of tags will be 933 and until if the condition $P_{\text {coll }}^{N, n}>P_{\text {coll }_{T h}}^{N, n}$ remains true the process continuous and remaining unidentified tags will be 733, 555, 366.

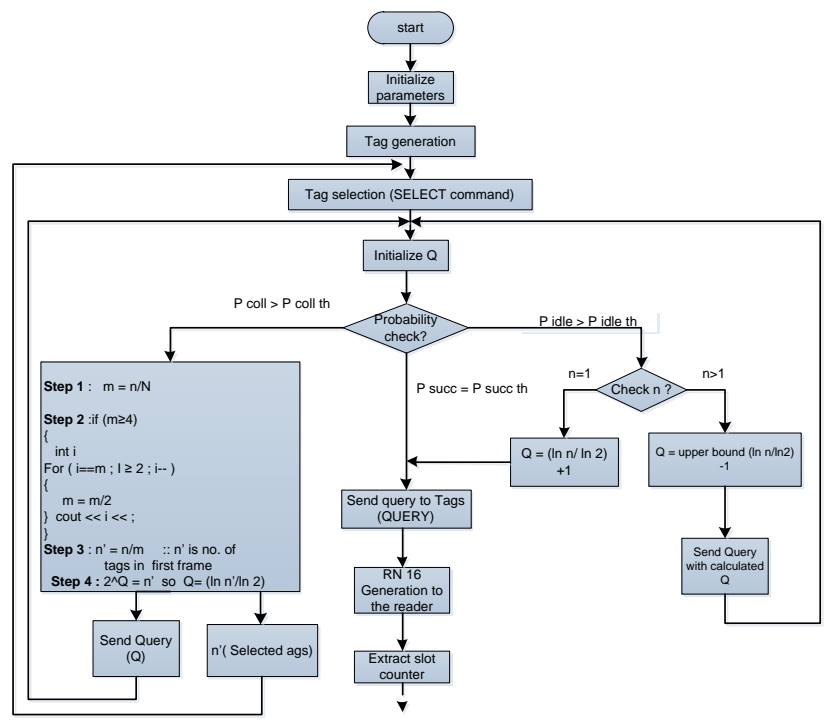

Figure 2: Probability check, frame sizing and dynamic tag grouping by Reader.

As, condition $P_{i d l e}^{N, n}>P_{i d l e_{T h}}^{N, n}$ will become true and the algorithm will find the most suitable frame size for these number of tags by using following relation.

$$
Q=\left\lceil\frac{\ln n}{\ln 2}\right\rceil-1=\left\lceil\frac{\ln 366}{\ln 2}\right\rceil-1=8.0
$$

So, the next frame size will be adjusted as $2^{8}=256$, but as number of tags is greater than frame size so condition $P_{\text {coll }}^{N, n} P_{\text {coll }_{T h}}^{N, n}$ will become true and reader will again divide tags by repeating all the four steps and divide the tags in groups with number of tags equal to 256 (which will participate in the current frame) and 220 which will come in the next frame. The remaining number of unread tags which will be identified due to the tags that participated in the current frame will be 162.

In the next read cycle as the number of tags is smaller compared to frame size so condition $P_{\text {idle }}^{N, n}>P_{\text {idl } e_{T h}}^{N, n}$ will become true and new frame size using equation (12) will be decided and sent by the reader to all the tags which will be $\mathrm{N}=128$ so again condition $P_{\text {coll }}^{N, n}>P_{\text {col } l_{T h}}^{N, n}$ will become true and remaining unread tags will be 81 .The process continuous till all the tags have been successfully identified. 


\subsubsection{Tag identification using Q-algorithm}

Figure 3 shows the process of tag identification using Qalgorithm. If the condition $P_{\text {succ }}=P_{\text {Suc }}$ bh becomes true only than the reader sends Query to all the tags. The tags select a slot randomly and the tag with slot number $=0$ replies back to the reader with RN16. Here as $\mathrm{n}=\mathrm{N}$ so there is minimum possibility of collision and slot to be idle. The reader upon receiving the tags response reply back with an ACK and tag than sends its EPC with CRC16 to the reader. The reader may end the frame by sending Query Adjust and if there are more tags to be identified in a frame than reader issues Query Reply till all the tags in the frame have been identified. After sending the Query Adjust command with $(\mathrm{Q}=\mathrm{Q})$ the reader broadcasts this Query command to all remaining unread tags and for which the probability condition is checked and suitable frame sizes for tag groups are decided by reader as discussed in Section B.

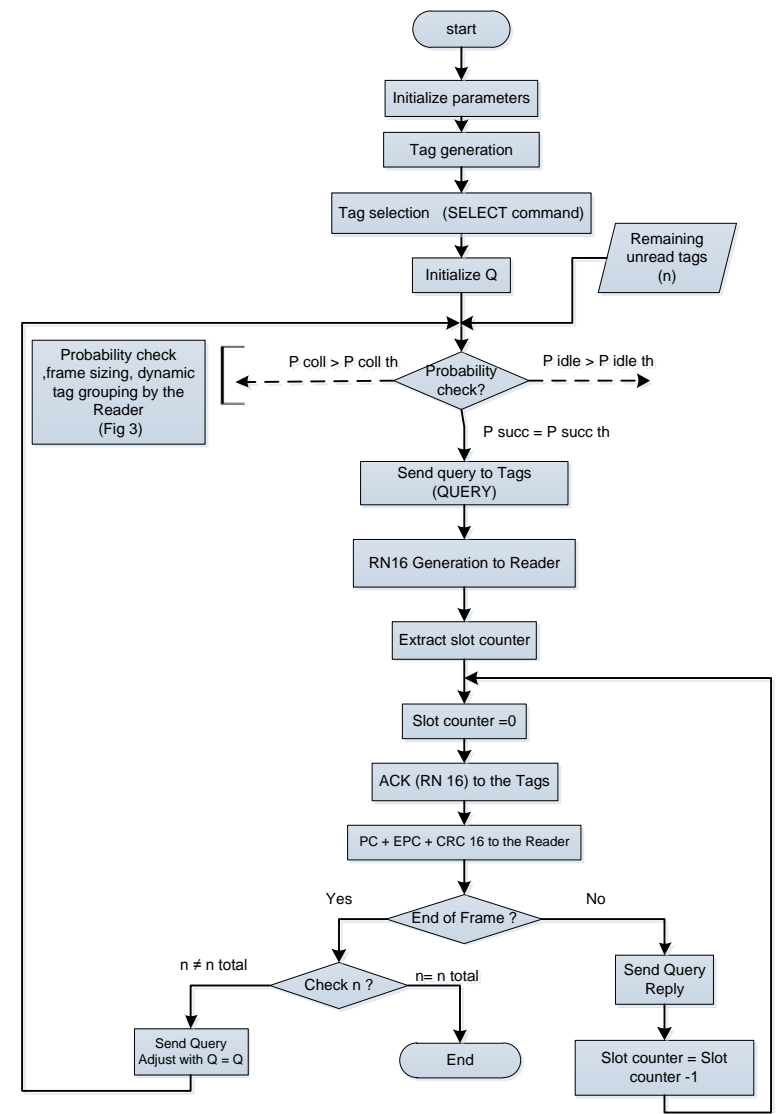

Figure 3: Tag identification using Q-algorithm for DGFSA

\section{PERFORMANCE ANALYSIS OF DGFSA}

This section shows the performance of DGFSA by varying number of tags with frame size $(\mathrm{N}=256)$ as shown in table 1 . The above table shows that as the number of tags varies with respect to frame size, DGFSA divides the tags in to number of groups keeping the system efficiency optimal. Thus the total number of slots required to identify the tags will vary linearly with respect to frame size.

\begin{tabular}{|l|l|l|l|l|l|l|l|}
\hline $\mathrm{n}$ & G1 & G2 & G3 & G4 & G5 & $:$ & $:$ \\
\hline 100 & 76 & 52 & 40 & 28 & 22 & $:$ & $:$ \\
\hline 200 & 153 & 106 & 82 & 58 & 46 & $:$ & $:$ \\
\hline 300 & 206 & 159 & 112 & 88 & 64 & $:$ & $:$ \\
\hline 400 & 306 & 212 & 165 & 118 & 94 & $:$ & $:$ \\
\hline 500 & 406 & 312 & 218 & 171 & 124 & $:$ & $:$ \\
\hline$:$ & $:$ & $:$ & $:$ & $:$ & $:$ & $:$ & $:$ \\
\hline 2300 & 1923 & 1546 & 1169 & 792 & 603 & $:$ & $:$ \\
\hline 2400 & 2023 & 1646 & 1269 & 892 & 703 & $:$ & $:$ \\
\hline 2500 & 2123 & 1746 & 1369 & 992 & 803 & $:$ & $:$ \\
\hline
\end{tabular}

TABLE 1 Tag division in frames (groups)

Figure 4 shows the behavior of DGFSA with other algorithms that how many slots are required to identify all the tags.

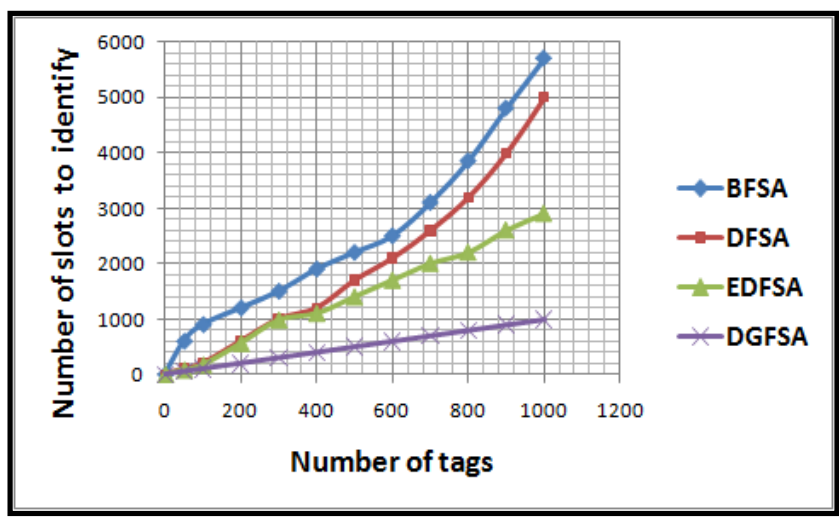

Figure 4: Number of Slots needed to identify tags

Figure 5 shows the collision ratio comparison of DGFSA with other algorithms with $\mathrm{N}=256$. It is seen that the graph line for DGFSA follows the optimal value line for collision ratio (0.2640).The graph for DFSA mostly remains below the optimal line while EDFSA graph varies along with increasing number of tags.

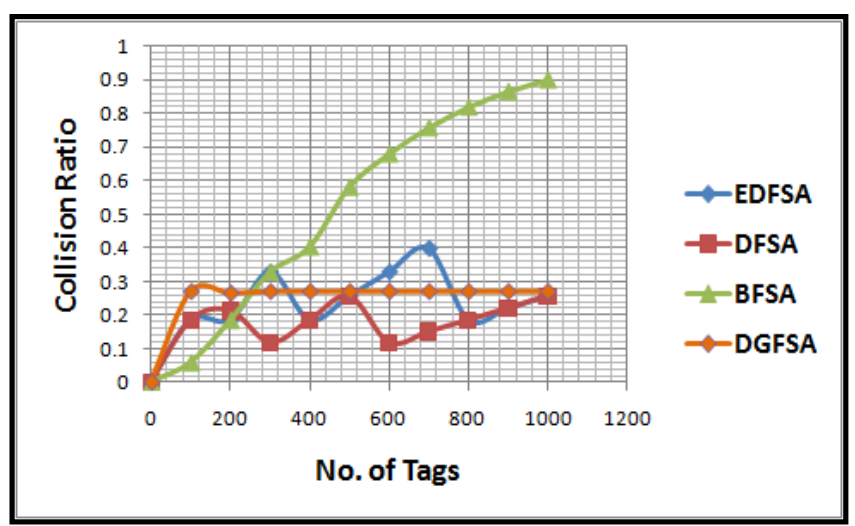

Figure 5: Collision ratio comparisons

DGFSA is built on the basis in order to reduce the identification time along with improvement in the system efficiency compared to the traditional anti collision algorithms. The system efficiency of DGFSA, as we vary the number of tags with respect to frame size is shown in Figure 
6. The system efficiency is always maintained to 0.368 which is the optimal system efficiency for Aloha based systems.

The identification time needed to identify all the tags is also reduced; from Figure1 in step 2.In order to reduce the number of inventory rounds DGFSA limits the number of frames, so it saves identification time.

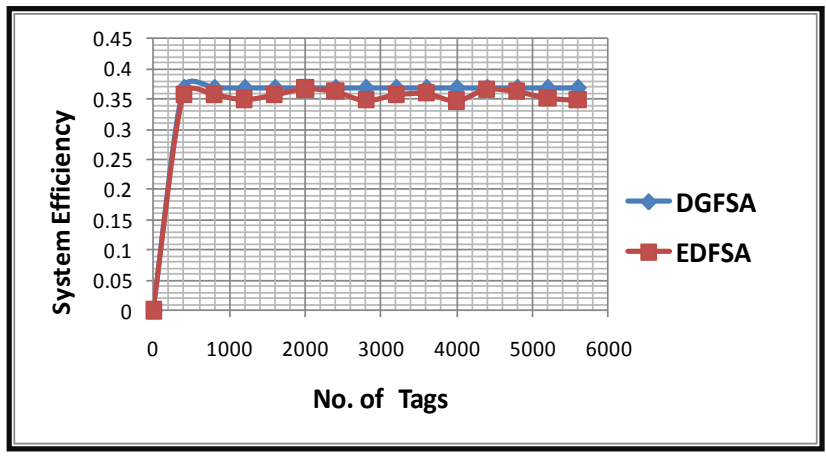

Figure 6 : System Efficiency of DGFSA ( $=256)$

Figure 7 shows that as the number of tags keep on increasing, the gap between the DGFSA and EDFSA is also increasing while for DFSA, it's making sinusoidal wave form.

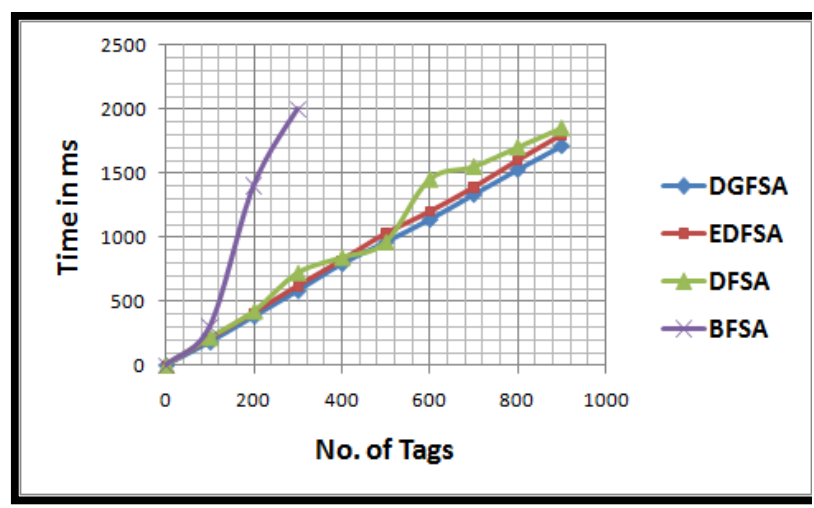

Figure 7 : Identification time results comparison.

\section{CONCLUSION}

Conventional Aloha based RFID anti collision algorithms mainly follow two techniques to identify the tags whether they may adjust the frame size or they may use static grouping approach with approach of grouping first and then identifying the tags group by group. Our algorithm is purely based on Gen-2, Q-protocol and uses Dynamic grouping approach in which tags are grouped and identified at the start of every frame. This approach out performs all other methods by dividing number of tags in to optimal number of groups with optimal frame size such that $(\mathrm{n}=\mathrm{N})$.
The algorithm has also improved identification time compared to other algorithms by saving inventory rounds and idle slots which are wasted in Q-algorithm in Gen2, which provided the maximum system efficiency. The probability approach provides a chance to distinguish them. This algorithm might be used in EPC-global Class1 Gen.2 to improve the performance with a little protocol change.

\section{REFERENCES}

[1] K.Finkenzeller and R. Waddington, RFID Handbook: Radio-Frequency Identification Fundamentals and Applications, John Wiley \& Sons, January2000. EPCglobal, EPC. Radio-frequency identity protocols class-1 generation- 2 UHF rfid protocol for communications at $860 \mathrm{MHz}-960 \mathrm{MHz}$ version technology.Version1.2.0,

2008.http:॥www.epcglobalinc.org\standards\

[2] Dongwook Lee, Okkyeong Bang, Sooyeol Im, and Hyuckjae Lee "Efficient Dual Bias Q-Algorithm and Optimum Weights for EPC Class1 Generation 2Protocol", Wireless Conference, 2008. EW 2008. 14th European. 07/2008; DOI: 10.1109/EW.2008.4623846; 2008

[3] Donghwan Lee, Kyungkyu Kim, Wonjun Lee, “ Q+Algorithm :An Enhanced RFID Tag Collision Arbitration Algorithm," Ubiquitous Intelligence and Computing: Lecture Notes in Computer Science,vol.4611/2007, pp.23-32, Aug. 2007.,2008

[4] Harald vogt-.Efficient object identification with passive RFID tags. In international conference on pervasive computing,LNCS.Springer-Verlag,2002

[5] H. Vogt, "Multiple object identification with passive RFID tags," 2002 IEEE International Conference on Systems, Man and Cybernetics, Oct. 2002

[6] C. Floerkemeier, "Bayesian transmission strategy for framed ALOHA based RFID protocols ," 2007 IEEE International Conference on RFID,March 2007

[7] Su-Ryun Lee, Sung-Don Joo, and Chae-Woo Lee, "An Enhanced Dynamic Framed SlottedALOHA Algorithm for RFID Tag Identification," The Proceeding of the 2nd Annual InternationalConference on Mobile and Ubiquitous Systems, 2005.

[8] Xu Huang, “An Improved ALOHA Algorithm for RFID Tag Identification," Lecture Notes in Computer Science. Springer Berlin/ Heideberg, Volume 4253/2006

[9] "Knowledge-Based Intelligent Information and Engineering Systems," KES06/ISBN 3-540-46535-9,B Gabrys, R.J.Howlett and L.C. Jain (Eds), pp1157-1162 2006 Springer-Verlag Berlin Heidelberg 2006Global generation 2 Protocol. Page 43. 